\title{
Design and Implementation about Secure Smart Electricity Meter Sealing Based on RF Tag
}

\author{
Zhenliu Zhou $^{1}$ \\ ${ }^{1}$ Shenyang Key Laboratory of Information Security for Power System, Institute of \\ Engineering, Shenyang, China \\ *Corresponding author: zhouzl@sie.edu.cn
}

\begin{abstract}
Smart electricity meter sealing plays a key role in the automation and test management to electricity smart meter device. Compared with traditional lead sealing and twodimensional code sealing, advantages of smart electricity meter sealing based RF tags include more convenient, more intelligent, more automated, and more secure. A new kind of smart electricity meter sealing based on RF tag is designed and produced, which conforms to meter device identify specification drafted by State Grid Corporation. Data structures, communication protocols, and secure mutual authentication protocol about this sealing are described. Particularly, this mutual authentication protocol is suitable for low-cost RF tags, and meets the State encryption control requirements for commercial encryption products. This sealing can provide integrity and confidentiality protection properties, prevents unauthorized access and tampering, and avoids illegal cloning.
\end{abstract}

Keywords: radio frequency identification; meter sealing; security; authentication protocol

\section{Introduction}

State Grid Corporation raised the demand for meter device "automated verification, intelligent warehousing, logistics of distribution," and meter service site management. With the advance of meter intensification and provincial measurement center construction, Standardized and automated meter sealing system efficiency has become a constraining factor. Currently, there are large differences in the types of products, materials, self-locking structure and encoding to different types of sealing. In order to promote the standardization of the electricity meter sealing, to improve the quality of meter sealing and the capability of electricitystealing prevention, it is necessary to conduct applied research on smart electricity meter sealing $[1,2]$.

Taking RF tags as electricity meter sealing is an emerging technology [3]. Radio frequency identification is a non-contact automatic identification technology, which automatically identifies the target object through the RF signal and access to relevant data, working without human intervention. It can work in a variety of harsh environments. RFID technology can identify fast moving objects, and can also identify multiple tags, it is easy to operate. In accordance with the operating frequency between tag and reader, it is divided into low-frequency, high frequency, ultra high frequency, etc. The vast majority of existing tags works in $13.56 \mathrm{MHz}$, while the passive UHF radio frequency identification (UHF RFID)working at 915 $\mathrm{MHz}$, for its long-range, high speed and low cost advantages of technology, has become a hot topic of RFID technology and will become commonplace for future. UHF RFID tags are used in this study, which are compliance with ISO/IEC 18000-6 Type $\mathrm{C}$ air interface requirements for passive backscatter tags. 
RFID systems can not protect the data stored in the RF tags if lacking of reliable security mechanisms. Others threats to RF tags include leaking sensitive information to the neighboring readers, easily susceptible to being interfered and being tracked $[8,9]$. Secure authentication protocols and privacy are the two key security issues when using RFID systems. There are many authentication protocols and privacy protection methods have been proposed aimed to RFID system, the typical protocols include Hash-based protocols [10,11], the digital library protocol [12], protocol based on the hash and change of tags ID [13], distributed RFID challenge-response protocol [14], protocol based on key array [15], protocol based on the LPN problem [16], as well as a variety of other suitable low-cost RFID privacy and authentication methods [17-21].

In this paper, Principles using RF tags as smart electricity meter sealing are proposed, data structures and communication protocols of this sealing are devised, finally, a kind of mutual authentication protocol based RFID system is implemented for low-cost RFID, which comply with the safety requirements of the State Grid Corporation of China.

\section{Design Principles of Smart Electricity Meter Sealing}

On the premise of meeting the existing functionality and business needs of meter work, the structural design of the RFID sealing must consider these aspects such as practicability, efficiency, reliability, and security.

(1) Practicability

First, this new RFID sealing must meet the existing business needs, reduce procurement costs and management costs for meter sealing, being conducive to its mass production and centralized bidding, ensure its successful implementation and application.

(2) Efficiency

Traditional pass-sealing exist some defects. It is difficult to automate sealed, the sealed procedure is inefficient seal, and sealed success rate is low. Therefore, new meter sealing and automatic sealed machine must be designed to overcome above shortcomings of traditional pass-sealing, improve sealed efficiency and success rate without affecting the tempo of the pipeline to meet the provincial center automation test meter, intelligent warehousing and logistics and distribution needs.

(3) Reliability

Reliability of sealing directly affects the use of meter device, reliable sealing will be helpful to improve the capability of electricity-stealing prevention, especially for the cover sealing in the terminal end.

\section{(4) Security}

Security Properties required for this RFID sealing include integrity, confidentiality, prevents unauthorized access and tampering, and difficult to illegal cloning. Compared to traditional lead sealing, RFID sealing has better controllability and security, More suitable for meter center to achieve centralized management, unified procurement, unified deployment, and to achieve the meter sealing life-cycle management in the end.

\section{Data Structures of Smart Meter Sealing}

RF tag memory area should include 64 bits as the reserved area (Reserved area), no less than 128 bits as Electronic Product Code (EPC area), 64 bits as the tag 
identifier area(TID area), and no less than 512 bits as user data area (User area). The data format in RF tags as a poter meter sealing is shown in Table 1.

Table 1. Data Format of RF Tags as Electricity Meter Sealing

\begin{tabular}{|c|c|}
\hline Area & Legnth(Bytes) \\
\hline Reserved Area & 8 \\
\hline EPC Area & $\geq 16$ \\
\hline TID & 8 \\
\hline User Data & $\geq 64$ \\
\hline
\end{tabular}

The RF tag should support ISO/IEC 18000-6 Type C all mandatory commands and access instruction. The RF tag should meet the requirements of ISO/IEC 180006 Type $\mathrm{C}$ multi-tag anti-collision.

\subsection{Reserved Area}

Reserved area is used for storing tags kill password and access password. Kill password and access password can provide authentication for the tags destruction and access. RF tag passwords must be different from each other. All passwords should be produced by the key management system of the State Grid Corporation.

Reserved area data storage format should follow the relevant requirements in ISO/IEC 18000-6 Type C.

\subsection{Electronic Product Code Area}

EPC Area is used for storing the unified code of the meter device. To modify data in this area, access password must be authenticated. The unified code of the meter device is stored as compressed BCD coded, and the start address of this area is $20 \mathrm{~h}$. The structure of EPC Area is shown in Table 2.

Table 2. Data Format of EPC Area

\begin{tabular}{|l|l|l|}
\hline Item Name & Memory Address & Data Description \\
\hline Version & Last two bits & $\begin{array}{l}\text { Version of the unified code of the meter } \\
\text { device }\end{array}$ \\
\hline Unified code & Start at $20 \mathrm{~h}$ & The unified code of the meter device \\
\hline $\begin{array}{l}\text { Control } \\
\text { Protocol }\end{array}$ & $10 \mathrm{~h}-1 \mathrm{Fh}$ & $\begin{array}{l}\text { Compliance with ISO/IEC 18000-6 Type C } \\
\text { standard }\end{array}$ \\
\hline CRC-16 & 00h-0Fh & $\begin{array}{l}\text { Data validation, compliance with ISO/IEC } \\
18000-6 \text { Type C standard }\end{array}$ \\
\hline
\end{tabular}

\subsection{Tag Identifier Area}

TID is the globally unique tag identifier for the tag, which is stored in TID area. It is permanent unchangeable. Its format must follow the relevant requirements in ISO/IEC 18000-6 Type C standard.

\subsection{User Data Area}

User data area is used to storing business data and related information about the electricity meter device. Access password must be authenticated to read or modify 
data in this area. All business data in this area are stored as compressed BCD code, ant the start address of business data is $30 \mathrm{~h}$.

Required contents of the user data area are recommended as Table 3.

Table 3. Data Format of User Data

\begin{tabular}{|l|l|l|}
\hline Item Name & Memory Address & Data Description \\
\hline Business data & Start at $30 \mathrm{~h}$ & $\begin{array}{l}\text { business data and related information about the } \\
\text { electricity meter device }\end{array}$ \\
\hline Data length & $20 \mathrm{~h}-2 \mathrm{Fh}$ & Length of business data \\
\hline MAC & $00 \mathrm{~h}-1 \mathrm{Fh}$ & $\begin{array}{l}\text { Tamper-resistant security authentication } \\
\text { information }\end{array}$ \\
\hline
\end{tabular}

In data item MAC, the tamper-resistant security authentication information is stored, which is automatic generated by the key management information systems of the State Grid Corporation.

The data item Data length stores the length of business data, which identifies the business data length in bits.

\subsection{Security}

RF tags must have the necessary security features. Data in EPC area and User data area must be protected for their integrity and Confidentiality to avoid unauthorized person illegally writing and tampering. RF tags should avoid being illegal cloned.

Access password of RF tags must be locked or un-locked, function of kill itself must be haven aroused by kill password. These two functions must comply with the related requirements of ISO/IEC 18000-6 Type C specification.

\section{Communication Protocols of Smart Electricity Meter Sealing}

Communication protocols about RFID system using smart electricity meter sealing must meet the related requirements of ISO/IEC 18000-6 Type C specification and requirements of specification [2007] No. 205 for RFID air Interface issued by Ministry of Information Industry.

\subsection{Communication Frame Format}

Communication Frame Format is defined to read/write data in RF tags.

Frame formats about reading tags are described as Table 4 and Table 5.

Frame formats about writing tags are described as Table 6 and Table 7.

Table 4. Frame Format to Read Tags

\begin{tabular}{|c|c|c|c|c|c|c|}
\hline $\begin{array}{c}\text { Command } \\
\text { Identifier }\end{array}$ & $\begin{array}{c}\text { Command } \\
\text { Code }\end{array}$ & $\begin{array}{c}\text { Access Area } \\
\text { Code }\end{array}$ & $\begin{array}{c}\text { Address } \\
\text { Pointer }\end{array}$ & $\begin{array}{c}\text { Data } \\
\text { Length }\end{array}$ & $\begin{array}{c}\text { Random } \\
\text { number }\end{array}$ & Checksum \\
\hline Lengths(bits) & 8 & 2 & 8 & 8 & 16 & 16 \\
\hline Description & 11000010 & $\begin{array}{c}\text { 00:reserved } \\
\text { area } \\
01: \text { EPC area } \\
10: \text { TID area } \\
\end{array}$ & $\begin{array}{c}\text { Pointer to } \\
\text { start } \\
\text { address } \\
\text { area } \\
\text { area }\end{array}$ & $\begin{array}{c}\text { Lengths } \\
\text { in bytes }\end{array}$ & handler & \\
& & & & & \\
& & & & & \\
\hline
\end{tabular}


Table 5. Frame Format of Successful Reading

\begin{tabular}{|c|c|c|c|c|}
\hline $\begin{array}{c}\text { Command } \\
\text { Identifier }\end{array}$ & $\begin{array}{c}\text { Command } \\
\text { Code }\end{array}$ & Data & $\begin{array}{c}\text { Random } \\
\text { number }\end{array}$ & Checksum \\
\hline Lengths(bits) & 1 & $\begin{array}{c}\text { Variable- } \\
\text { length }\end{array}$ & 16 & 16 \\
\hline Description & 0 & Return data & handler & \\
\hline
\end{tabular}

Table 6. Frame Format to Write Tags

\begin{tabular}{|c|c|c|c|c|c|c|}
\hline $\begin{array}{c}\text { Command } \\
\text { Identifier }\end{array}$ & $\begin{array}{c}\text { Comman } \\
\text { d Code }\end{array}$ & $\begin{array}{c}\text { Access } \\
\text { Area Code }\end{array}$ & $\begin{array}{c}\text { Address } \\
\text { Pointer }\end{array}$ & data & $\begin{array}{c}\text { Random } \\
\text { number }\end{array}$ & Checksum \\
\hline $\begin{array}{c}\text { Lengths(bits } \\
\text { ) }\end{array}$ & 8 & 2 & 8 & 16 & 16 & 16 \\
\hline Description & 11000011 & $\begin{array}{c}\text { 00:reserved } \\
\text { area } \\
\text { 01:EPC area } \\
\text { 10:TID area } \\
\text { 11:User } \\
\text { data area }\end{array}$ & $\begin{array}{c}\text { Pointer to } \\
\text { start } \\
\text { address }\end{array}$ & $\begin{array}{c}\text { Data to } \\
\text { write }\end{array}$ & handler & \\
& & & & \\
\hline
\end{tabular}

Table 7. Frame Format of Successful Writing

\begin{tabular}{|c|c|c|c|}
\hline $\begin{array}{c}\text { Command } \\
\text { Identifier }\end{array}$ & $\begin{array}{c}\text { Command } \\
\text { Code }\end{array}$ & $\begin{array}{c}\text { Random } \\
\text { number }\end{array}$ & Checksum \\
\hline Lengths(bits) & 1 & 16 & 16 \\
\hline Description & 0 & handler & \\
\hline
\end{tabular}

\subsection{RF Conformance Requirements}

The RF performance meet the requirements with ISO/IEC 18000-6 Type C in section 9.3, these requirements include frequency, channel, demodulation capabilities, encoding formats, RF envelope, frequency hopping, emission mask, and so on.

\subsection{Protocol Conformance Requirements}

The protocol meet the requirements with ISO/IEC 18000-6 Tyoe C in Section 9.2, these requirements include time parameters, response status of tags, data of tags, and so on.

\section{Secure Mutual Authentication Protocol for Smart Electricity Meter Sealing}

To promote security of RFID system for using smart electricity Meter Sealing, follows should be done:

(1) To prevent illegal readers get the tags information or modify the tags data, and to prevent counterfeit tags deceive readers, mutual authentication mechanism among tags and readers should be adopted.

(2) Using cipher-text in communication process. Appropriate encryption algorithms should be selected because of features of low cost, low power consumption and less computing and memory resource of RF tags.

(3) In addition to communications among readers and tags, back-end database management in the security of the RFID system has also played a crucial role. This 
part if attacked will cause the system to leak a large number of tags data, cipher keys and other information. So it is necessary to strengthen the security of the backend database management.

RF tag communicates with readers through the built-in antenna. If the tag antenna is connected with the metallic packaging of the product, then the RF tag is formed integrally with the product. In the process of production, using technical process to make the tag and the product can not be separated. This will make the product difficult to forge.

The new smart electricity meter sealing is shown in figure 1. National encryption algorithm SM7 is adopted in this sealing. The algorithm is recognized by the State Encryption Administration, specially designed for electronic tags using for commercial encryption algorithm, its key length is 128 bits.
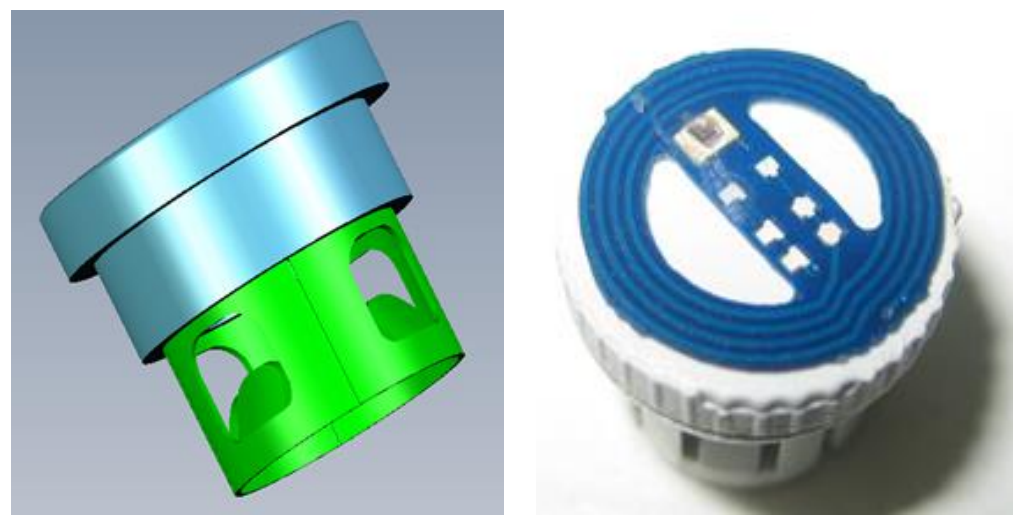

Figure 1. Smart Electricity Meter Sealing Packed with RF Tag

\subsection{Procedure of Mutual Authentication}

When using SM7 algorithm for mutual authentication, the procedure between the sealing and the reader can be divided into two steps. The first step, the reader sends an authentication request, the sealing returns a random number (denoted as RT). The second step, the reader sends the cipher-text Token1, the sealing decrypts Token1, and compare with RT, if matched, then sends the cipher-text Token2, the reader decrypt Token2, and compare with RR, if matched, then the procedure of this authentication is finished. This procedure is brief descried as Figure 2.

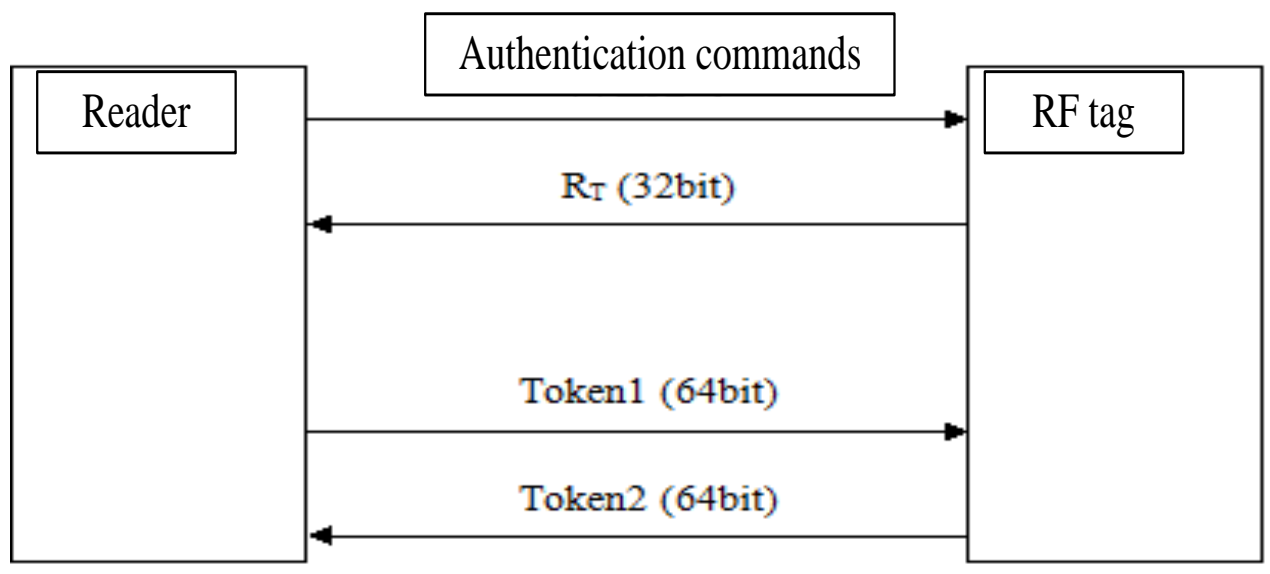

Figure 2. Procedure of Mutual Authentication 
Detailed procedure is described as follows.

(1) The reader sends authentication request.

(2) The random number generator in the sealing generates a 32 bits random number RT. The sealing sends RT to the reader.

(3) The reader generates a 32bits random number RR after receiving RT, encrypts $\mathrm{RR} \| \mathrm{RT}$ into Token 1 using algorithm SM7, sends Token 1 to the sealing.

(4) The sealing receives Token 1 and encrypts it, gets the plaintext high 32 bits content as RR' and low 32 bits content as RT'. Comparing RT' with RT, if RT' is equal to RT, the sealing generates a new random number RT', encrypts RT'||RR'into Token 2 using algorithm SM7, sends Token2 to the reader. Meanwhile, the sealing takes Token2 as IV(defined in 5.2), takes current SM7 key as block cipher key, conducts SM7 block cipher encryption to produce key stream Ci required by stream cipher. If RT' is not equal to RT, the authentication failed.

(5) The reader receives Token 2 and decrypts it, gets the plaintext low 32 bits content as RR'. Comparing RR' with RR, if RR' ' is equal to RR, the authentication succeeded. Meanwhile, the reader takes Token2 as IV, takes current SM7 key as block cipher key, conducts SM7 block cipher encryption to produce key stream Ci required by stream cipher. If RR" is not equal to RR, the authentication failed.

\subsection{Principle of Generating a Key Stream of stream Cipher through Block Cipher Algorithm SM7}

The key stream for stream cipher is produced through block cipher algorithm SM7, the operation is as follows:

(1) The first operation: P0 = IV , C0 = RFIDSF_E[KEY]( P0)

IV: the encryption result Token 2 produced during above procedure of a mutual authentication.

KEY: the 128 bits cipher key of SM7 used for a mutual authentication

(2) The second and subsequent operation: $\mathrm{Pi}=\mathrm{Ci}-1, \mathrm{Ci}=$ RFIDSF_E[KEY]( Pi) $(\mathrm{i} \geq 1)$

Sequence $\mathrm{Ci}(\mathrm{i} \geq 0)$ is just the key stream for stream cipher generated by block cipher algorithm SM7.

(3)Encryption of stream cipher: $\mathrm{Ei}=\mathrm{Ci} \oplus \mathrm{Di}$

When using a stream key to encrypt plaintext Di, making Di XOR Ci to obtain cipher text Ei, lower bit of sequence $\mathrm{Ci}$ is used firstly.

\section{(4)Decryption of stream cipher: $\mathrm{Di}{ }^{\prime}=\mathrm{Ci} \oplus \mathrm{Ei}$}

When using a stream key to decrypt cipher text Ei, making Ei XOR Ci to obtain plaintext Di', lower bit of sequence Ci is used firstly.

The sealing and the reader should use the same sequence $\mathrm{Ci}$, and maintain synchronization of $i$. If any errors occur in an encrypted communication, the current key stream $\mathrm{Ci}$ should be stopped using, and a new key stream $\mathrm{Ci}$ ' should be recalculated.

\subsection{Precautions for using SM7 Algorithm}

When using SM7 algorithm to encrypt communication data, the ISO14443 TypeA chip can be used in the following two ways: 
(1) All the communication data are stream encrypted at the physical layer, including valid data, parity, CRC, and etc.

(2)Only valid data are stream encrypted, others data at the physical layer such as parity and CRC are not encrypted.

Which encryption way is selected is determined by the highest bit of the byte value SAK that is returned by command Select, if 0 , the first encryption way is selected, if 1 , the second encryption way is selected.

While the ISO14443 TypeB chip is used for encryption communication, there are the same two encryption way for selecting. Which encryption way is selected is determined by the highest bit of the nibble value Protocol_Type that is returned by command REQB/WUPB/Slot-MARKER, if 0, the first encryption way is selected, if 1 , the second encryption way is selected.

During the stream encrypted communication, if an authentication is required again, the first authentication command should continue to being encrypted transmitted, and all following response commands and data are transmitted normally without stream cipher.

\section{Conclusion}

This new kind of RFID-based smart electricity meter sealing can be used to replace current lead sealing, it is more smart and secure, facilitates the management of smart electricity meter, meets the test requirements for pipeline, has the capability of electricity-stealing prevention. Currently in some areas (such as Wuhan), this new kind of sealing has begun small-scale, small-batch trial use. But the higher price compared with lead sealing impacts of its wide application. In addition, for this kind of smart meter sealing, the RFID system has integrity and confidentiality protection capabilities, can avoid unauthorized read, write, tampering and recognition, but in terms of dealing with the physical cloning of RF tag (including RF tag TID clones, although doing so costly, but still be possible), there is still no proper solution.

\section{Acknowledgements}

This research is supported by Liaoning BaiQianWan Talents Program ((No.2013921051), and science and technology research program of Liaoning Province Education Administration (No.L2013493). Special thanks for above supports very much.

\section{References}

[1] W. N. Wang, Z. Q. Wang and S. Yang, "Technical Analysis about Electricity-stealing and ElectricityStealing Prevention Aimed at Smart Power Meter", Hunan Electric Power, vol. 32, no. 30, (2012).

[2] Y. Y. Lai, "Prevention Methods about Power Stealing aimed at Electronic Power Meter", Public Communication of Science and Technology, vol. 4, no. 173, (2011).

[3] L. Q. Wang, Z. Y. Zan, L. J. Ding, X. Li and Y. Ling, "Study on Smart Seal Technology based on RF tag", Water Resources and Power, vol. 10, no. 183, (2012).

[4] S. E. Sarma, S. A. Weis and D. W. Engels, "RFID systems and security and privacy implications", Cryptographic Hardware and Embedded Systems-CHES 2002. Springer Berlin Heidelberg, vol. 454, (2003).

[5] S. E. Sarma, S. A. Weis and D. W. Engels, "Radio frequency identification: Secure Risks and challenges", RSA Laboratories Cryptobytes, vol. 6, no. 2, (2003).

[6] S. A. Weis, S. E. Sarma and R. L. Rivest, "Security and privacy aspects of low-cost radio frequency identification systems", Security in pervasive computing. Springer Berlin Heidelberg, vol. 201, (2004).

[7] Y. B. Zhou and D. G. Feng, "Design and Analysis of Cryptographic Protocols for RFID", Chinese Journal of Computers, vol. 29, (2006), pp. 581.

[8] L. Adam, founder, The Bunker. Practical attacks against RFID, Network Security, vol. 9, (2007). 
[9] A. Mitrokotsa, M. R. Rieback and A. S. Tanenbaum, "Classifying RFID attacks and defenses", Information Systems Frontiers, vol. 12, no. 491, (2010).

[10] M. Ohkubo, K. Suzuki and Ki noshi ta S. Hash, "Chain based forward secure Privacy Protection scheme for low cost RFID", Proceedings of the 2004 Symposium on Cryptography and Information Security(SCIS2004), Sendai, vol. 719, (2004).

[11] D. Henrici and P. Muller, "Hash-based enhancement of location privacy for radio-frequency identification devices using varying identifiers", Pervasive Computing and Communications Workshops, 2004. Proceedings of the Second IEEE Annual Conference, IEEE, vol. 149, (2004).

[12] D. Molnar and D. Wagner, "Privacy and security in library RFID: issues, practices, and architectures", Proceedings of the 11th ACM conference on Computer and communications security, ACM, vol. 210, (2004).

[13] H. Zhang, C. H. Hou and D. H. Wang, "A New Security and Privacy on RFID Mutual Authentication Protocol Based on Partial ID”, Journal of Electronics \& Information Technology, vol. 31, no. 853, (2009).

[14] K. Rhee, J. Kwak and S. Kim, "Challenge-response based RFID authentication protocol for distributed database environment", Security in Pervasive Computing. Springer Berlin Heidelberg, vol. 70, (2005).

[15] Z. G. Ding, L. Guo and Y. J. Wang, "An Authentication Protocol Based on Key Array for RFID", Journal of Electronics \& Information Technology, vol. 31, no. 722, (2009).

[16] J. Tang and D. Y. Ji, "Design and Analysis of Security Protocols for RFID Based on LPN Problem", Journal of Electronics \& Information Technology, vol. 31, no. 439, (2009).

[17] Y. W. Wang, Y. H. Zhao and X. C. Li, "RFID Authentication Scheme Based on EPC-C1G2 Standard", Computer Engineering, vol. 36, no. 153, (2010).

[18] A. Juels, "Minimalist cryptography for low-cost RFID tags", Security in Communication Networks, Springer Berlin Heidelberg, vol. 149, (2005).

[19] A. Juels, R. L. Rivest and M. Szydlo, "The blocker tag: selective blocking of RFID tags for consumer privacy", Proceedings of the 10th ACM conference on Computer and communications security, ACM, vol. 103, (2003)

[20] D. Benessa, F. Kevin and J. Ari, “Cryptanalysis of Two Lightweight RFID Authentication Schemes”, In International Workshop on Pervasive Computing and Communication Security-PerSec, vol. 211, (2007).

[21] J. Bringer, H. Chabanne and T. Icart, "Cryptanalysis of EC-RAC, a RFID identification protocol", Cryptology and Network Security, Springer Berlin Heidelberg, vol. 149, (2008).

\section{Author}

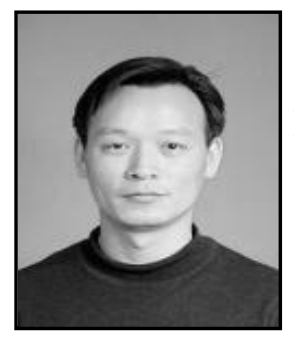

Zhenliu Zhou, (1971-), male, the Han nationality, native place: Hubei, Associate Prof. of Computer Science, Tel: 86-024-31975614, E-mail: zhouzl@sie.edu.cn. 
International Journal of Security and Its Applications

Vol. 9, No. 6 (2015) 